\title{
Transmission of Neisseria gonorrhoeae among men who have sex with men: an anatomical site-specific mathematical model and impact of mouthwash
}

\author{
Lei Zhang ${ }^{1-4}$, David G Regan5, Eric PF Chow ${ }^{1-2}$, Manoj Gambhir ${ }^{3}$, Vincent Cornelisse ${ }^{1-2}$, Andrew Grulich ${ }^{5}$, \\ Jason Ong ${ }^{1-2}$, David A. Lewis ${ }^{6-7}$, Jane Hocking ${ }^{8}$, Christopher K Fairley ${ }^{1-2}$
}

1. Melbourne Sexual Health Centre, Alfred Health, Melbourne, Australia; 2. Central Clinical School, Faculty of Medicine, Monash University, Melbourne, Australia; 3. School of Public Health and Preventive Medicine, Faculty of Medicine, Monash University, Melbourne, Australia; 4. Research Centre for Public Health, Tsinghua University, Beijing 100084, China; 5. Kirby Institute, UNSW Australia, Sydney, Australia; 6. Marie Bashir Institute for Infectious Diseases \& Biosecurity and Sydney Medical School-Westmead, University of Sydney, Sydney, Australia; 7. Western Sydney Sexual Health Centre, Western Sydney Local Health District, Parramatta, Australia; 8. Melbourne School of Population Melbourne Sexual and Global Health, Melbourne University, Melbourne, Australia

\section{AIM}

Gonorrhoea notifications are rapidly rising in men who have sex with men (MSM). We developed a mathematical model to assess mouthwash as a novel intervention for gonorrhoea control.

\section{METHODS}

- Compartmental model of Neisseria gonorrhoeae (NG) transmission to explain anatomic site-specific prevalence of gonorrhoea among MSM.

- The model was calibrated to available epidemiological (Melbourne sexual health centre) and behavioural data (systematic review).

- Estimate per-act transmission of NG using a MonteCarlo approach

- Evaluate the potential impacts of screening scale-up and utilisation of mouthwash on the gonorrhoea epidemic.

\section{RESULTS}

1. Empirical prevalence of oropharyngeal, anal and urethral gonorrhoea of $10.6 \%(8.1-12.2 \%), 8.6 \%$ $(6.7-10.4 \%)$ and $0.20 \% \quad(0.04-0.35 \%)$ among MSM.

2. Oropharynx to oropharynx transmission through kissing $73.6 \%[63.7-78.4 \%]$ of gonorrhoea incident cases in MSM.

3. Increasing annual oropharynx screening for gonorrhoea from the current $40 \%$ to $100 \%$ may only halve the prevalence of gonorrhoea in MSM.

4. Use of mouthwash with moderate efficacy (additional 1\% clearance per daily use) would further reduce the oropharyngeal, anal and urethral prevalence rates to $2.1 \%(1.3 \%-2.3 \%), 2.2 \%$ $(1.5-2.6 \%)$ and $0.06 \%(0.03-0.10 \%)$,

5. A high efficacy mouthwash (additional 1.5\% clearance per daily use) may reduce gonorrhoea prevalence close to elimination.

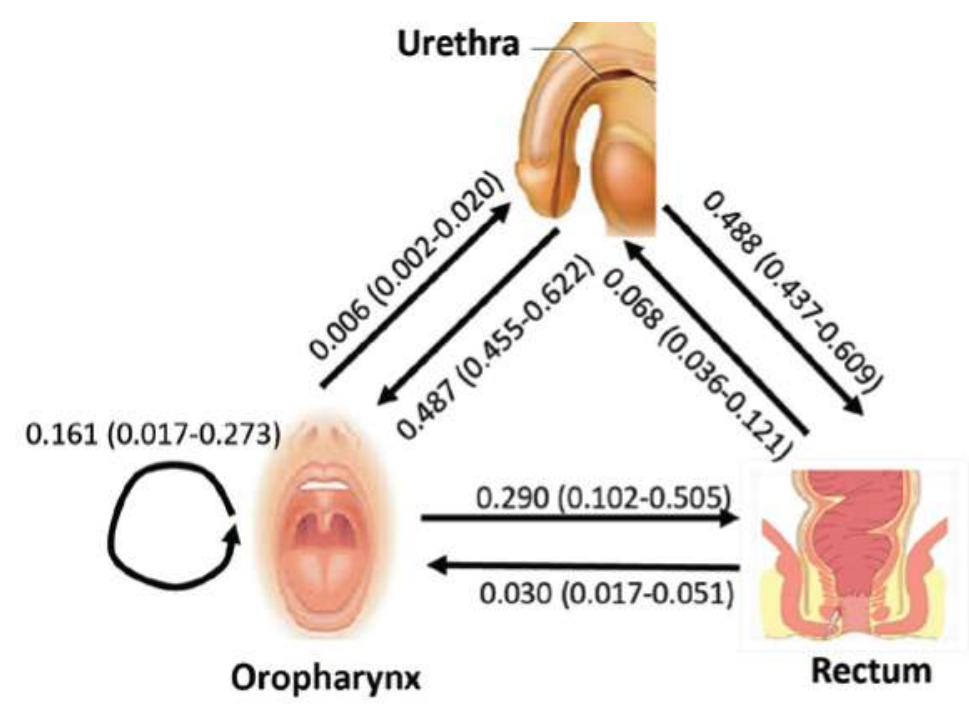

Figure 2. Incidence and composition of gonorrhoea incident cases by route of transmission

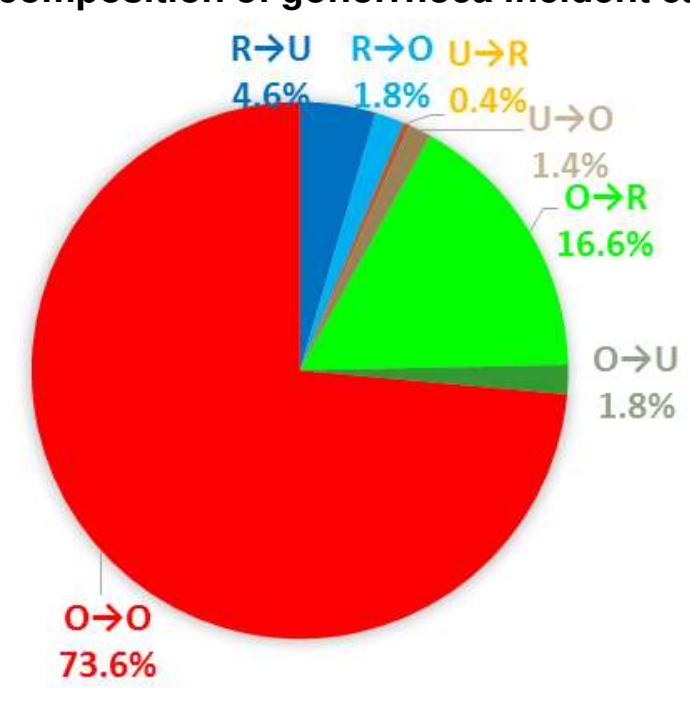

O: oropharyngeal R: rectum

Figure 3. Reduction in gonorrhoea prevalence at anatomical sites by increasing screening or mouth rinse
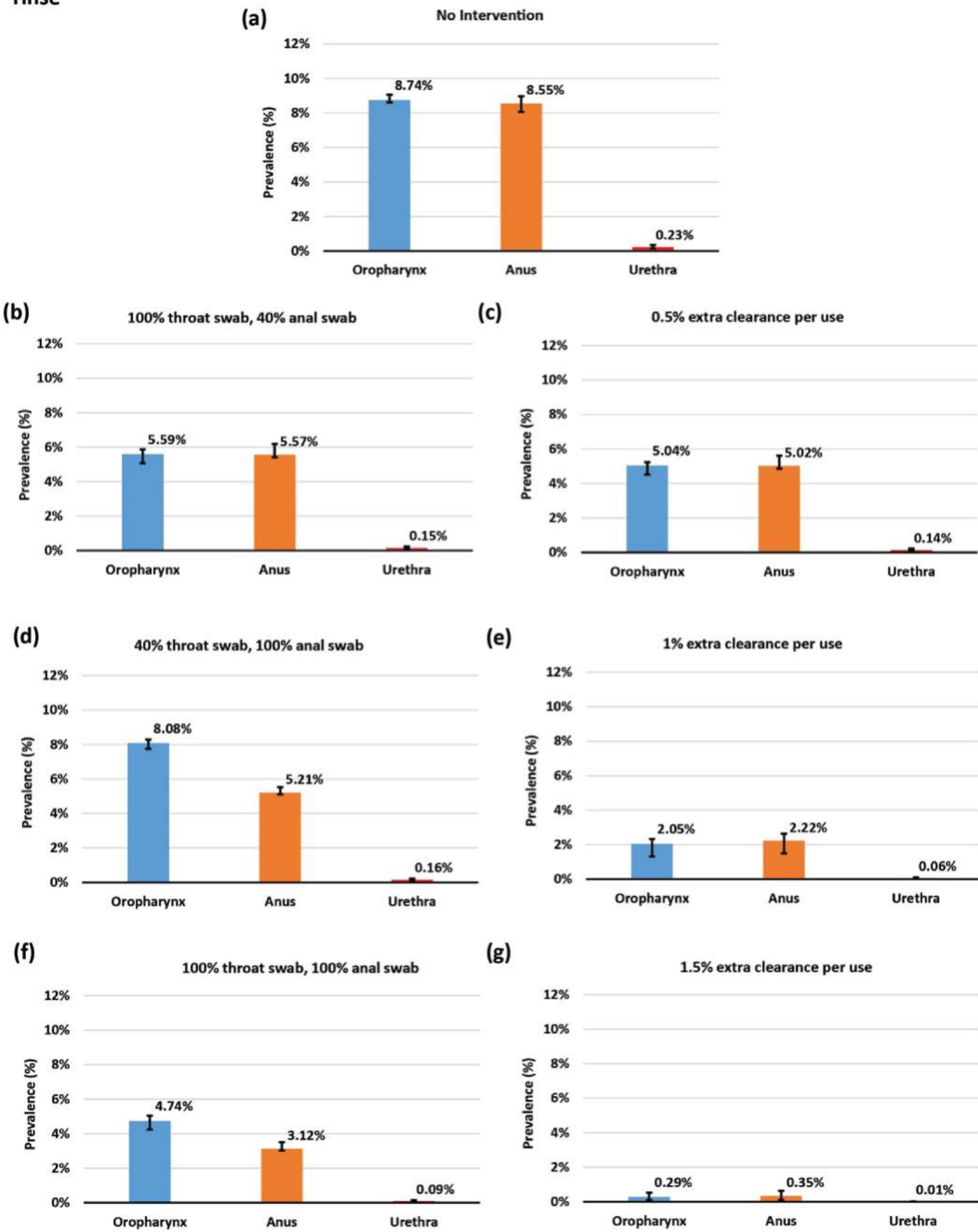

$(g)$

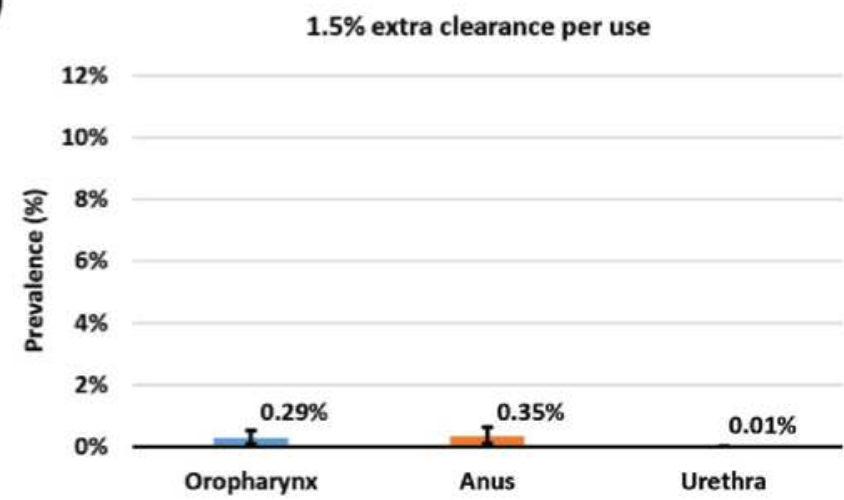

CONCLUSIONS

- Kissing may play a key role in NG transmission among MSM.

- STI screening alone is insufficient to control the rising epidemic.

- Promotion of regular mouthwash may achieve near elimination of gonorrhoea in MSM.
CONTACT DETAILS

A/Prof Lei Zhang, Melbourne Sexual Health Centre, 580 Swanston Street, Carlton, VIC 3053, Australia, Email: lei.zhang1@mshc.org.au 